\title{
Polymorphisms of Plasminogen Activator Inhibitor- I 4G/5G, Coagulation Factor XIII Val34Leu and Angiotensin Converting Enzyme I/D Impact on Recurrent Implantation Failure
}

\begin{abstract}
Recurrent implantation failure (RIF) is recognized when failure of transferred embryos to implant occurs following repeated in vitro fertilization (IVF) cycles. During cytotrophoblast invasion, plasminogen activator inhibitor 1 (PAI-1) appears to be involved in controlling proteolysis and maternal tissue remodeling. Coagulation factor XIII (FXIII) may have an impact on the ability of the trophoblast to invade into the endometrium and to stabilize attachment with fibrin crosslinking. Angiotensin converting enzyme (ACE) participates in the regulation of vascular tone and changes in vascular metabolites affect the functions of the fetoplacental complex and may induce abnormalities of blood circulation in the placenta. The aim of the present work was to compare the predictive value of PAI-1 4G/5G, FXIII Val34Leu and ACE I/D polymorphisms on the IVF outcome in women with RIF. The present study was conducted on 60 women with RIF (RIF group) and 60 healthy, age-matched women eligible for IVF with positive $\beta$-human chorionic gonadotropin ( $\beta$-HCG) two weeks after embryo transfer (control group). Polymerase chain reaction-restriction fragment length polymorphism (PCR-RFLP) for the PAI-1 4G/5G, FXIII Val34Leu and ACE I/D polymorphisms was performed for all participants. The RIF group showed higher predominance of the $4 \mathrm{G}$ allele of the PAI-1 polymorphism - $6754 \mathrm{G} / 5 \mathrm{G}(\mathrm{P}=0.029)$. Higher frequencies of the heterozygous and homozygous FXIII polymorphism were noted in the RIF group ( $\mathrm{P}=0.016,0.020$ respectively) together with higher risk to carry the Leu allele $(\mathrm{P}=0.001)$. The heterozygous $\mathrm{ACE}$ I/D polymorphism was predominant in the RIF group $(\mathrm{P}=0.011)$. It could be concluded that certain studied polymorphisms (PAI-1 4G/5G and the FXIII Val34Leu, and to a lesser extent the ACE I/D) could be associated with repeated implantation failure.
\end{abstract}

Keywords: plasminogen activator inhibitor 1, coagulation factor XIII, angiotensin converting enzyme, gene, polymorphism, recurrent implantation failure
Volume 6 Issue I - 2018

\author{
Hadeer A Abbassy, ' Ahmed F Galal, ${ }^{2}$ Ashraf \\ H Abdelrahman, ${ }^{2}$ \\ 'Department of Clinical pathology, Alexandria University, Egypt \\ ${ }^{2}$ Department of Obstetrics \& Gynecology, Alexandria University, \\ Egypt
}

Correspondence: Hadeer A Abbassy, Clinical pathology department, Faculty of Medicine,Alexandria University, Ahmed Yehia street, Zezenia, Alexandria, Egypt, Fax +20358502 10, Tel +201066751862, Email hadeerabbassy@gmail.com

Received: November 22, 2017 | Published: January 19, 2018
Abbreviations: RIF, recurrent implantation failure; IVF, in vitro fertilization; PAI-1, plasminogen activator inhibitor 1; FXIII, coagulation factor XIII; ACE, angiotensin converting enzyme; $\beta$-HCG, $\beta$-human chorionic gonadotropin; PCR-RFLP, polymerase chain reaction restriction fragment length polymorphism

\section{Introduction}

Recurrent implantation failure (RIF) is recognized when transferred embryos fail to implant following repeated in vitro fertilization (IVF) cycles. ${ }^{1}$ RIF is commonly defined as the failure of implantation after three or more consecutive IVF attempts, with which 1-2 high-grade embryos were transferred in each cycle. ${ }^{2}$ Cytotrophoblast invasion to the proper depth of the uterus secures proper anchorage for the conceptus which is needed for successful implantation. ${ }^{3}$ A functional balance of fibrinolysis and coagulation may secure adequate trophoblast invasion, which is crucial for a regular implantation. ${ }^{4}$ During cytotrophoblast invasion, plasminogen activator inhibitor 1 (PAI-1) appears to be involved in controlling proteolysis and remodeling of maternal tissue. ${ }^{5}$ PAI-1 inhibits tissueand urokinase-type plasminogen activators, thus decreasing plasmin production and the dissolution of fibrin clots. The PAI-1 gene is located on the long arm of chromosome 7 (7q21.3-q22). Plasma PAI-1 levels are linked with a common guanosine insertion/deletion gene polymorphism, 4G/5G, 675 bp upstream from the start site of translation and higher levels have been associated with homozygosity for the deletion genotype $(4 \mathrm{G} / 4 \mathrm{G})$ than those associated with the insertion genotype $(5 \mathrm{G} / 5 \mathrm{G})$, and in turn with impaired fibrinolytic activity. ${ }^{6} \mathrm{PAI}-1$ on one hand, inhibits urokinase plasminogen activator (uPA)-urokinase plasminogen activator receptor (uPAR) leading to inhibition of trophoblast invasion, ${ }^{7}$ and on the other, it may trigger or augment the trophoblast invasion process. ${ }^{8}$ However, an association has been found between PAI-1 $-6754 \mathrm{G} / 5 \mathrm{G}$ polymorphism and recurrent pregnancy loss (RPL) ${ }^{9}$ and to a lesser extent RIF. ${ }^{10}$ Coagulation factor XIII (FXIII) covalently cross-links fibrin and affects fibrinolysis, thus it may have an impact on the ability of the trophoblast to invade into the endometrium and to stabilize attachment with fibrin cross-linking. FXIII Val34Leu polymorphism in exon 2 of the FXIII gene is correlated with earlier cross-linking, a finer fibrin meshwork formation, and lower susceptibility to fibrinolysis. ${ }^{11}$ This polymorphism has been reported to carry an elevated overall risk for RPL in women homozygous for the FXIII 34Leu. ${ }^{12}$ 
Angiotensin converting enzyme (ACE) is a dipeptidyl carboxypeptidase which is encoded by the ACE gene, located on chromosome $17 \mathrm{q} 23$ and contains 26 exons and 25 introns. ${ }^{13}$ The ACE insertion (I)/deletion (D) polymorphism was strongly associated with the level of the circulating enzyme, where the D allele was associated with an elevated level. ${ }^{14}$ The ACE participates in the regulation of vascular tone and changes in vascular metabolites were found to affect the functions of the fetoplacental complex and may induce abnormalities of blood circulation in the placenta, resulting in RPL. ${ }^{15}$

The aim of this work was to assess the predictive value of the polymorphisms of PAI-1 4G/5G, FXIII Val34Leu and ACE I/D on the IVF outcome in Egyptian women with repeated IVF failure. They were compared to healthy age-matched women eligible for IVF with positive $\beta$-HCG were selected two weeks after embryo transfer as a control group. The end-point of the study was diagnosis of biochemical pregnancy.

\section{Materials and methods}

Between October 2014 and June 2016, the current study was conducted on 60 women indicated for in vitro fertilization/ intracytoplasmic sperm injection (IVF/ICSI) treatment. They were selected from the IVF unit, department of Obstetrics and Gynecology at Shatby University Hospital, Alexandria University. The evolution of pregnancy was not recorded. Sixty healthy, age-matched women eligible for IVF with positive $\beta$-HCG were selected two weeks after embryo transfer as a control group. They had no personal history of thrombotic or bleeding disorder or any history of known hereditary or acquired thrombophilic alteration and without any personal history of miscarriage. The protocol of the study was approved by the institutional ethics committee and was in accordance to the commitment of the Helsinki declaration. All participants provided informed written consent before inclusion in the study.

\section{Exclusion criteria related to both the RIF and control groups}

Women less than 20years or above 37 years of age, with a body weight less than $50 \mathrm{~kg}$ or more than $100 \mathrm{~kg}$, with a personal or family history of hereditary or acquired thrombophilia, or venous thromboembolism (VTE), or under active anticoagulant or antiplatelet treatment, or using these agents during the last 30 days before inclusion. Women with abnormal complete blood count or platelet count and ongoing cardiovascular, renal or liver disease, known systematic or chronic disease (autoimmune syndrome, heart disease, severe or uncontrolled thyroid disease or HIV infection), arterial hypertension, malignancy, or ovarian insufficiency (FSH 9IU/ml and/or number of antral follicles 8 ) or polycystic ovary syndrome (defined according to the Rotterdam criteria). Women under treatment with non-steroid anti-inflammatory drugs within the last 10days before inclusion.

\section{Methodology}

This prospective controlled study included 120 women indicated for IVF/ICSI treatment after RIF. All couples underwent a standard infertility evaluation that included a baseline transvaginal ultrasonography, evaluation of tubal patency either by hysterosalpingography (HSG) or laparoscopy, a semen analysis using WHO criteria, and a baseline hormonal profile that included FSH, LH, TSH, and PRL in the early follicular phase. All couples were counseled about the risks and benefits of enrollment in the study. Patients who have agreed to continue with ICSI were divided into two groups, 60 with history of recurrent ICSI failure and 60 as a control group without prior history of ICSI treatment. Selected women in both groups had their blood count, platelet count, prothrombin time, activated partial thromboplastin time, fibrinogen, renal and liver functions within the normal range.

To induce controlled ovarian stimulation, estrogen production was initially downregulated using either a long gonadotropin-releasing hormone (GnRH) agonist or a short agonist or an antagonist protocol. Ovarian stimulation was done using recombinant human follicular stimulating hormone (FSH) in addition to human menopausal gonadotropins (HMG) at doses ranging from $75 \mathrm{IU}$ to $450 \mathrm{IU}$ per day according to the patient age, body mass index (BMI), antral follicle count, size and number of follicles and estradiol concentrations (E2). This stimulation was initiated once pituitary desensitization had been achieved (E2 concentration $<50 \mathrm{pg} / \mathrm{mL}$ ). The response was followed by E2 measurement six days later and by ultrasound scanning of the ovarian follicles at days 9-10 after the first FSH injection, and repeated when necessary. Patient monitoring was done by repeated vaginal ultrasound examinations and the dose of FSH/HMG was tailored depending on their response and continued until the day of human recombinant chorion gonadotrophin (hCG) administration. When at least 3 follicles reached $17 \mathrm{~mm}$ in diameter, a single injection of HCG (10000IU) was administered. Transvaginal oocyte retrieval was scheduled 34 to $36 \mathrm{~h}$ after HCG injection and performed under vaginal ultrasound guidance.

Then a semen sample was requested from the male partner and assessed for sperm concentration and motility. ${ }^{16}$ The most motile spermatozoa were selected by performing a sperm gradient technique with double sperm wash or by a swim-up procedure. Embryo transfer was performed 72hours after oocyte retrieval. On day 2 , individually cultured embryos were evaluated on the basis of the number of blastomeres, blastomere size, fragmentation rate and presence of multinucleated blastomeres. Therefore, the oocytes were retrieved 10 to 14 days after starting the stimulation with the FSH. Two or three embryos were transferred 72hours after oocyte retrieval into the uterine cavity under abdominal ultrasound guidance. Daily vaginal progesterone administration in a dose of $400 \mathrm{mg}$ twice daily was used for luteal phase support from the day of oocyte retrieval until the day of pregnancy test (Prontogest 400mg suppositories, IBSA, Cairo, Egypt). A pregnancy test was done 14days after oocyte retrieval and when the serum beta-HCG concentration was over $5 \mathrm{IU} / 1$, it was considered positive. An early trans-vaginal scan was performed 4weeks after embryo transfer for women with a positive result. A clinical pregnancy was identified by the presence of a viable intrauterine gestational sac at 6-8 weeks with a pulsating heart.

\section{Blood sampling}

Five $\mathrm{ml}$ of venous blood was collected from each participant. Blood was stored in sterile EDTA vacutainer tube at $-20^{\circ} \mathrm{C}$ for genomic DNA extraction. Patients were subjected to routine investigations, including complete blood picture, prothrombin time, international normalization ratio (INR) and activated partial thromboplastin time (aPTT) and assay of protein C, protein S, antithrombin III and lupus anticoagulant. PCR-RFLP for the PAI-1 4G/5G, FXIII Val34Leu and ACE I/D polymorphisms was done for RIF cases and the control group.

\section{Detection of PAI-I, FXIII and ACE polymorphisms by PCR-RFLP}

Genomic DNA was isolated from peripheral blood leucocytes using Wizard genomic DNA purification kit (Promega, Madison, WI, USA), according to the manufacturer's protocol. 
The PAI-1 -675 4G/5G polymorphism was detected using forward (5'-CCA ACA GAG GAC TCT TGG TC-3') and reverse (5'-CAC AGA GAG AGT CTG GCC ACG-3') primers.

The FXIII Val34Leu polymorphism was detected using forward (5'-CAT GCC TTT TCT GTT GTC TTC-3') and reverse (5'-TAC CTT GCA GGT TGA CGC CCC GGG GCA CTA-3') primers. The ACE I/D polymorphism was detected using forward (5'-CTG GAG ACC ACT CCC ATC CTT TCT-3') and reverse (5'-GAT GTG GCC ATC ACA TTC GTC AGA T-3') primers.

PCR was performed using DNA thermocycler (PTC-100 programmable thermal controller; MJ Research, Watertown, Massachusetts, USA). The PCR mixture consisted of $1.5 \mathrm{mM} \mathrm{MgCl}_{2}$, $200 \mu \mathrm{M}$ dNTPs, $0.4 \mu \mathrm{M}$ of each primer, $1 \mathrm{U}$ Taq DNA polymerase, and $25 \mathrm{ng}$ genomic extracted DNA in a reaction volume of $25 \mu \mathrm{L}$. The DNA was denatured at $94^{\circ} \mathrm{C}$ for $5 \mathrm{~min}$, followed by amplification for 35 cycles of denaturation at $94^{\circ} \mathrm{C}$ for $30 \mathrm{~s}$, annealing at $58^{\circ} \mathrm{C}$ for 30 s, and extension at $72^{\circ} \mathrm{C}$ for 30 s. Final extension was conducted at $72{ }^{\circ} \mathrm{C}$ for $5 \mathrm{~min}$. DNA fragments were separated by $2 \%$ agarose gel electrophoresis and identified by ethidium bromide staining. After generation of the PCR product, restriction enzymes were added and incubated at $37^{\circ} \mathrm{C}$ for $15 \mathrm{~min}$. for the detection of the PAI- $14 \mathrm{G} / 5 \mathrm{G}$, FXIII Val34Leu and ACE I/D polymorphisms using agarose gel electrophoresis and ultra-violet light illumination.

\section{Statistical analysis}

Data was analysed using IBM SPSS advanced statistics version 20 (SPSS Inc., Chicago, Illinois, USA). Numerical data were expressed either as mean and standard deviation or as median and range. Qualitative data were expressed as frequency and percentage. The Kolmogorov-Smirnov and the Shapiro-Wilk tests were employed to identify parameters with skewed distribution. To examine the relation between qualitative variables, Chi-square test or Fisher's exact test was used. For quantitative normally distributed data, comparison between two groups was done using Student's t-test and comparison between three groups was done using Kruskal-Wallis test [nonparametric analysis of variance (ANOVA)]. Odds ratio (OR) with $95 \%$ confidence interval $(\mathrm{CI})$ was used for risk estimation. $\mathrm{P}$ value less than 0.05 was considered significant.

\section{Results and discussion}

The RIF and control groups matched in their age and BMI ( $p$ $0.05)$. Age at examination $(\mathrm{P}=0.290)$ and mean $\mathrm{BMI}(\mathrm{P}=0.353)$ were comparable between the RIF and control groups with no statistical significance.

\section{Results of PAI-I -675 4G/5G polymorphism}

The prevalence of PAI-1-675 4G/5G polymorphism in total study population was $(30 \%)$ for the heterozygous group and $(8.3 \%)$ for the homozygous group respectively. Meanwhile, the $4 \mathrm{G} / 5 \mathrm{G}$ polymorphism among the control population was (20\%) for the heterozygous group and $(5 \%)$ for the homozygous group respectively. Cases with RIF were more prone to carry the $4 \mathrm{G}$ allele $(\mathrm{P}=0.029)$, whereas no statistical significance was found regarding the heterozygous and homozygous polymorphisms between the RIF group and the control group ( $\mathrm{P}=0.2$ and 0.1 respectively) as shown in Table 1 .

Table I Plasminogen activator inhibitor- I 4G/5G polymorphism in recurrent implantation failure group $(\mathrm{N}=60)$ and control group $(\mathrm{N}=60)$

\begin{tabular}{llllll}
\hline & Wild-type & Heterozygous & Homozygous & 4G allele frequency & 5G allele frequency \\
\hline Cases & $37(61.7 \%)$ & $18(30 \%)$ & $5(8.3 \%)$ & $15(21.4 \%)$ & $55(78.6 \%)$ \\
Controls & $45(75 \%)$ & $12(20 \%)$ & $3(5 \%)$ & $17(12.9 \%)$ & $113(87.1 \%)$ \\
P value & 0.311 & 0.212 & 0.12 & $0.029 *$ & 0.421 \\
\hline
\end{tabular}

\section{Results of FXIII Val34Leu polymorphism}

As regards RIF cases with FXIII Val34Leu polymorphism, (21.6\%) were heterozygous and (11.7\%) were homozygous, whereas (10\%) of the control group were heterozygous and $(1.7 \%)$ were homozygous. RIF cases were more likely to be carriers of the heterozygous and homozygous FXIII polymorphism than the control group $(\mathrm{P}=0.016$, 0.020 respectively) as shown in Table 2 . This raises the potential that the heterozygous and homozygous FXIII polymorphism carriers are at higher risk to develop RIF. RIF cases were also found to be at higher risk to carry the Leu allele $(\mathrm{P}=0.001)$.

Table 2 Coagulation factor XIII Val34Leu polymorphism in recurrent implantation failure group $(N=60)$ and control group $(N=60)$

\begin{tabular}{llllll}
\hline & Wild-type & Heterozygous & Homozygous & Val allele frequency & Leu allele frequency \\
\hline Cases & $40(66.7 \%)$ & $13(21.6 \%)$ & $7(11.7 \%)$ & $54(77.1 \%)$ & $16(22.9 \%)$ \\
Controls & $53(88.3 \%)$ & $6(10 \%)$ & $1(1.7 \%)$ & $121(93.1 \%)$ & $9(6.9 \%)$ \\
P value & 0.151 & $0.016 *$ & $0.020^{*}$ & 0.187 & $0.001 * *$ \\
\hline
\end{tabular}

\section{Results of ACE I/D polymorphism}

The results concerning the ACE I/D polymorphism are presented in Table 3. RIF cases were (16.7\%) heterozygous for this polymorphism and $(20 \%)$ were homozygous, while $(6.7 \%)$ of the control group were heterozygous and (11.6\%) were homozygous. Cases with RIF were more liable to carry the heterozygous ACE polymorphism than the control group ( $\mathrm{P}=0.011)$. Cases with RIF were more likely to carry the $\mathrm{D}$ allele than the control group but there was no statistical significance $(\mathrm{P}=0.322)$.

Table 3 Angiotensin converting enzyme I/D polymorphisms in recurrent implantation failure group $(\mathrm{N}=60)$ and control group $(\mathrm{N}=60)$

\begin{tabular}{llllll}
\hline & Wild-type & Heterozygous & Homozygous & I allele frequency & D allele frequency \\
\hline Cases & $38(63.3 \%)$ & $10(16.7 \%)$ & $12(20 \%)$ & $20(28.6 \%)$ & $50(71.4 \%)$ \\
Controls & $49(81.7 \%)$ & $4(6.7 \%)$ & $7(11.6 \%)$ & $39(30 \%)$ & $91(70 \%)$ \\
P value & 0.201 & $0.011^{*}$ & 0.102 & 0.143 & 0.322 \\
\hline
\end{tabular}




\section{Discussion}

Fine-tuned endometrial vascular remodeling with adequate fibrinolysis is essential for a sufficient trophoblast invasion into maternal spiral arteries and thus development of a low-resistance uteroplacental circulation and successful implantation. ${ }^{17}$ As PAI$14 \mathrm{G} / 4 \mathrm{G}$, factor XIII V34L and ACE I/D polymorphisms interfere with fibrin crosslinking and regulation of fibrinolysis, they may be expected to contribute to RIF.

In the current study, no significant difference was found between RIF cases and the control group as regards both the heterozygous and homozygous PAI-1-675 4G/5G polymorphisms. However, it was reported that women with a history of implantation failure have displayed a significantly higher prevalence of PAI-1 4G/5G mutations than controls. ${ }^{18}$ Meanwhile, the RIF group was more prone to carry the $4 \mathrm{G}$ allele than the control group. This result agrees with another study which has detected an increased incidence of the $4 \mathrm{G}$ allele and/or 4G/4G genotype among the patients with RPL. ${ }^{10}$ Moreover, the prevalence of homozygous PAI-1 4G/4G polymorphism was demonstrated to be higher in patients with RIF and RPL. ${ }^{19}$ It was also reported that low molecular weight heparin and metformin have been demonstrated to improve implantation process and ameliorate pregnancy complications in patients with $4 \mathrm{G} / 4 \mathrm{G}$ genotype because of the hypofibrinolytic impact of overexpressed PAI-1 on implantation. ${ }^{20}$

The heterozygous and homozygous FXIII Val34Leu polymorphisms as well as the Leu allele frequency were significantly higher in the RIF group than the control group. This coincides with the results of a recent study which pointed out that heterozygosity and, to a slightly larger extent, homozygosity for FXIII Val34Leu polymorphism might be considered as risk factors for RPL. ${ }^{21}$ In addition, FXIII Val34Leu polymorphism has been reported to carry an elevated overall risk for RPL in women homozygous for the FXIII 34Leu as well as in compound carriers of the FXIII 34Leu. ${ }^{12}$

In the present study, the prevalence of the heterozygous ACE I/D polymorphism among patients with RIF was significantly more common than the control group. RIF cases were also more likely to carry the $\mathrm{D}$ allele than control group but the difference was not statistically significant. The D allele was reported to induce an elevated expression of PAI-1 which is hypofibrinolytic. ${ }^{22}$ Hence, the $\mathrm{D}$ allele may be predisposing to excess fibrin accumulations in spiral arteries and within the intervillous spaces that may impede perfusion and prevent implantation. ${ }^{23}$ Furthermore, another study demonstrated an association between ACE I/D polymorphism and risk of RPL. ${ }^{24}$ The ACE polymorphic D allele was also implicated as an increased risk factor for RPL. ${ }^{25}$

\section{Conclusion}

It could be concluded that certain studied polymorphisms (PAI-1 $4 \mathrm{G} / 5 \mathrm{G}$ and the FXIII Val34Leu, and to a lesser extent the ACE I/D) could be associated with implantation failure.

\section{Acknowledgements}

Dr Hadeer A. Abbassy designed the research study and wrote the paper. Dr Ahmed F. Galal clinically examined and treated the patients. Dr Hadeer Abbassy contributed essential laboratory reagents and techniques. Dr Ashraf H. Abdelrahman analysed the data.

\section{Conflict of interest}

The authors declare that they have no conflict of interest and no funding sources were available.

\section{References}

1. Simon A, Laufer N. Repeated implantation failure: clinical approach. Fertil Steril. 2012;97(5):1039-1043.

2. Simon A, Laufer N. Assessment and treatment of repeated implantation failure (RIF). J Assist Reprod Genet. 2012;29(11):1227-1239.

3. Feng Q, Liu Y, Liu K, et al. Expression of urokinase, plasminogen activator inhibitors and urokinase receptor in pregnant rhesus monkey uterus during early placentation. Placenta. 2000;21(2-3):184-193.

4. Dossenbach-Glaninger A, van Trotsenburg M, Oberkanins C, et al. Risk for early pregnancy loss by factor XIII Val34Leu: The impact of fibrinogen concentration. J Clin Lab Anal. 2013;27(6):444-449.

5. Floridon $\mathrm{C}$, Nielsen $\mathrm{O}$, Hølund B, et al. Does plasminogen activator inhibitor-1 (PAI-1) control trophoblast invasion? A study of fetal and maternal tissue in intrauterine, tubal and molar pregnancies. Placenta. 2000;21(8):754-762.

6. Margaglione M, Grandone E, Vecchione G, et al. Plasminogen activator inhibitor-1 (PAI-1) antigen plasma levels in subjects attending a metabolic ward: relation to polymorphisms of PAI-1 and angiontensin converting enzyme (ACE) genes. Arterioscler Thromb Vasc Biol. 1997;17(10):2082-2087.

7. Naruse K, Lash GE, Bulmer JN, et al. The urokinase plasminogen activator (uPA) system in uterine natural killer cells in the placental bed during early pregnancy. Placenta. 2009;30(5):398-404.

8. Labied S, Blacher $\mathrm{S}$, Carmeliet $\mathrm{P}$, et al. Transient reduction of placental angiogenesis in PAI-1-deficient mice. Physiol Genomics. 2011;43(4):188-198.

9. Li X, Liu Y, Zhang R, et al. Meta-analysis of the association between plasminogen activator inhibitor-1 $4 \mathrm{G} / 5 \mathrm{G}$ polymorphism and recurrent pregnancy loss. Med Sci Monit. 2015;21:1051-1056.

10. Khosravi F, Zarei S, Ahmadvand N, et al. Association between plasminogen activator inhibitor 1 gene mutation and different subgroups of recurrent miscarriage and implantation failure. J Assist Reprod Genet. 2014;31(1):121-124

11. Ariëns RA, Philippou H, Nagaswami C, et al. The factor XIII V34L polymorphism accelerates thrombin activation of factor XIII and affects cross-linked fibrin structure. Blood. 2000;96(3):988-995.

12. Dossenbach-Glaninger A, van Trotsenburg M, Dossenbach $M$, et al. Plasminogen activator inhibitor $14 \mathrm{G} / 5 \mathrm{G}$ polymorphism and coagulation factor XIII Val34Leu polymorphism: Impaired fibrinolysis and early pregnancy loss. Clin Chem. 2003;49(7):1081-1086.

13. Ibarra-Rubio ME, Pedraza-Chaverrí J. Renin: structure and expression regulation of the gene, biosynthesis, and cellular pathways of secretion. Rev Invest Clin. 1993;45:255-266.

14. Rigat B, Hubert C, Alhenc-Gelas F, et al. An insertion/deletion polymorphism in the angiotensin I-converting enzyme gene accounting for half the variance of serum enzyme levels. J Clin Invest. 1990;86(4):1343-1346

15. Mahendru AA, Everett TR, McEniery CM, et al. Cardiovascular function in women with recurrent miscarriage, preeclampsia and/or intrauterine growth restriction. J Matern Fetal Neonatal Med. 2013;26(4):351-356.

16. World Health Organization. WHO Laboratory Manual for the Examination and Processing of Human Semen. 5th ed. Geneva: World Health Organization; 2010.

17. Buchholz T, Lohse P, Rogenhofer N, et al. Polymorphisms in the ACE and PAI-1 genes are associated with recurrent spontaneous miscarriages. Hum Reprod. 2003;18(11):2473-2477.

18. Coulam CB, Jeyendran RS, Fishel LA, et al. Multiple thrombophilic gene mutations are risk factors for implantation failure. Reprod Biomed Online. 2006;12(3):322-327. 
19. Salazar Garcia MD, Sung N, Mullenix TM, et al. Plasminogen activator inhibitor-1 4G/5G polymorphism is associated with reproductive failure: metabolic, hormonal, and immune profiles. Am J Reprod Immunol. 2016;76(1):70-81.

20. Ivanov P, Tsvyatkovska T, Konova E, et al. Inherited thrombophilia and IVF failure: the impact of coagulation disorders on implantation process. Am J Reprod Immunol. 2012;68(3):189-198.

21. Seyed Mehdi Sajjadi, Abbas Khosravi, Jalil Pakravesh, et al. Factor XIII Val34Leu polymorphism and risk of recurrent pregnancy loss in Iranian population: a case control study. Frontiers in Biology. 2016;11(6):471475 .

22. Kim DK, Kim JW, Kim S, et al. Polymorphism of angiotensin converting enzyme gene is associated with circulating levels of plasminogen activator inhibitor-1. Arterioscler Thromb Vasc Biol. 1997;17(11):32423247.
23. Al-Mukaynizi FB, AlKhuriji A, Babay Z, et al. Lack of association between Angiotensin converting enzyme I/D polymorphism and unexplained recurrent miscarriage in Saudi Arabia. J Med Biochem. 2016;35(2):166-173

24. Jeon YJ, Kim JH, Lee BE, et al. Association between polymorphisms in the reninangiotensin system genes and prevalence of spontaneously aborted fetuses. Am J Reprod Immunol. 2013;70(3):238-245.

25. Wang Z, Wang $P$, Wang $X$, et al. Significant association between angiotensin-converting enzyme gene insertion/deletion polymorphism and risk of recurrent miscarriage: a systematic review and metaanalysis. Metabolism. 2013;62(9):1227-1238. 